\title{
Analysis Range-Free Node Location Algorithm in WSN
}

\author{
Xiaojun Liu ${ }^{1, a}$ and Jianyu Wang ${ }^{1}$ \\ ${ }^{1}$ School of Transportation Huanggang Normal University, Hubei Huanggang, China \\ awhutliuxiaojun@126.com
}

Keywords: DV-HOP; Centroid; Amorphous; APIT; Convex optimization; MDS-MAP.

\begin{abstract}
This paper firstly introduced the basic concept of node location algorithm in WSN, node location technology standard of performance evaluation. Then analyzed the localization process of the six range-free localization algorithms, performance, application scope and the problems. Finally, node density including network node and beacon node, positioning error and positioning precision were compared through the form.
\end{abstract}

\section{Introduction}

In the practical application of WSN, the sensor node location information has become one of the essential information in the whole network, many applications once lost the position information of nodes, the network will become useless, so the sensor network node location technology has become a important hot topic in the field of many scientists. There are many algorithms in the process of the realization of the positioning; according to different standards have different classified method. The classification of the most common is: Range-Based and Range-Free localization algorithm [1]. If localization algorithm needs to know the unknown node to the reference node or Beacon nodes the absolute distance between, then to calculate the unknown node coordinate information, such as locating methods can be called a Range - -based localization algorithm. On the other hand, the other algorithm without measuring the distance between the node value localization algorithms called Range - Free. Range-based on the precision of localization algorithm is superior to the Range - Free localization algorithm, but need to measure distance, relatively large amount of calculation, to spend a lot of energy, does not apply to low power consumption, low cost, and other areas of the application[2]. Range-Free localization algorithm because do not need to accurately measure the distance between nodes information, greatly reduces the requirement for the node hardware, and it is calculated using the estimated distance between nodes, so less affected by environment, in many of the node location accuracy requirements can be extensively used in WSN is not high.

\section{The Node Location Technology Performance Evaluation Standard}

In the wireless sensor network (WSN) positioning technology, different localization algorithm has different influence on positioning results, usually has the following several indicators to measure [3]:

Positional Accuracy: position precision is refers to the spatial location information (usually the coordinates) proximity between its true location, it is a leading indicator of measuring sensor network localization, only reach a certain position precision of localization algorithm is real and effective.

Effective Range of Orientation: positioning system can locate Effective range. To satisfy most of the nodes in WSN can be positioning, only covers a wide range of node location to be meaningful.

Node Density: refers to the spread of sensor Node Density in terms of network nodes. In WSN node density had a great influence on the performance of the positioning, usually Node Density high positioning precision will be higher and higher, the opposite will reduce Positional Accuracy. In view of the different localization algorithm in WSN node density is not required for the same, the performance of the sensor nodes and the price also determines the node of the seeding density. 
Density of Beacon Node: Density of Beacon Node refers to the Beacon nodes in the proportion in the WSN. Beacon node has its own positioning function, the price is more expensive, could not sow large area, its node determines the positioning precision of the high and low density.

Fault Tolerance and Adaptability: refers to the so-called fault-tolerance of the presence of Fault system will not fail, the characteristics of can still work normally. Fault Tolerance is Fault how should the Fault, to be exact and not let Error. Adaptability can be thought of as a can adjust itself according to environmental changes can intelligent features of feedback control system, so that the system can work in accordance with the standards of some setting in the optimal state.

Security: Security is refers to the system to legitimate users of response and to resist the illegal request, to protect themselves from the external influence and the ability to attack. WSN usually work in the area of the physical environment is more complicated, positioning system vulnerable to environmental or man-made destruction and attacks, which cannot reach the ideal wireless communication environment to achieve the positioning of the effect, therefore must have a very strong security positioning system and algorithm.

Power Dissipation: refers to the Power loss of Power consumption, Power consumption in the process of WSN design has always been one of the main aspects in its application. Limited due to the energy of the sensor nodes and it is not easy to get, so we need the whole WSN can with less energy consumption and high efficiency of energy utilization to implement security positioning is the first question faced by the current research.

Cost and Consideration: the cost of location algorithm includes time cost, capital cost and space cost. Under the premise of ensuring the accuracy of positioning, the positioning system should be the minimum cost, such as the amount of computation required, the amount of communication, storage space, etc.

\section{The Main Range-Free Localization Algorithm Analysis}

Although positioning algorithm based on distance measurement can realize accurate positioning, but also often higher requirements for hardware, hardware cost increasing, is not conducive to the use of large area, in various fields, wide Angle. Without ranging localization algorithm is simple to use, only need to use the unknown node to estimate the distance between the reference node or other location information, and then use Trilateration or Maximum Likelihood Method to calculate the unknown node coordinate information. The existing positioning algorithm based on without range mainly include: DV - HOP algorithm, Centroid algorithm, amorphous algorithm, APIT algorithm, Convex Optimization algorithm and MDS-MAP algorithm [4-5], etc.

DV-HOP Algorithm. DV-HOP algorithm does not require network nodes have distance function, which USES the average of the jump distance to represent the distance between the adjacent nodes, to calculate the actual distance, the coordinates are calculated by the trilateral measurement method. Algorithm of faults is to use the jump distance between the two nodes represent the actual linear distance will cause the error, the accuracy of positioning. Therefore, DV-HOP algorithm in the high density of nodes in the network can achieve high precision positioning, positioning accuracy is not ideal in sparse network.

Centroid. Centroid refers to the physical system is considered to focus on the quality of an imaginary point. In sensor networks, the center of mass is to point to the center of the polygon, the polygons each vertex coordinates of the geometric average is the bar centric coordinates. Centroid algorithm is the University of Southern California's Nirupama Bulusu etc, the algorithm is suitable for outdoor positioning under the circumstance of high node density, and it is based on the network connectivity (Connectedness) implementation of node localization. Its basic idea is: the unknown nodes according to their own set of threshold method to receive the beacon nodes around the contains the node ID and position information to determine its area, according to its beacon node ID and position information received by the calculation area in the center of mass, and the area of the center of mass as its own positioning results. This algorithm is simple, the hardware requirements is low, don't 
need any additional hardware devices, but in the process of positioning requires more beacon nodes, and the algorithm are assumed in the process of locating nodes with wireless signal transmission model of the ideal, this also has the very big difference with the actual situation, thus affecting the positioning precision of the Centroid. The basic principle of centroid is shown in Fig. 1.

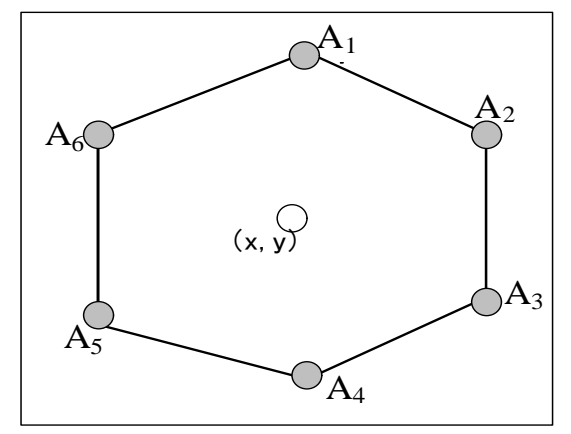

Figure 1. Diagram centroid algorithm

In this algorithm, $A_{1} 、 A_{2} 、 A_{3} \cdots A_{6}$ Six vertex coordinates are: $\left(x_{1}, y_{1}\right),\left(x_{2}, y_{2}\right),\left(x_{3}, y_{3}\right),\left(x_{4}, y_{4}\right)$, $\left(x_{5}, y_{5}\right) 、\left(x_{6}, y_{6}\right)$, the area of the center of mass as follows:

$$
(x, y)=\left(\frac{x_{1}+x_{2}+x_{3}+x_{4}+x_{5}+x_{6}}{6}, \frac{y_{1}+y_{2}+y_{3}+y_{4}+y_{5}+y_{6}}{6}\right)
$$

Amorphous. Amorphous is based on the connectivity of the localization algorithm; it needs to know in advance the connectivity of the network. Algorithm implementation process, the unknown node first calculated with the minimum hop count between each beacon node; And then calculate the unknown node to jump distance of each beacon node, which assumes that the network node communication radius is the same; Finally re-use Trilateration or MLE algorithm for position calculation. Amorphous is easier to implement, but the positioning precision is not high, there are two significant drawbacks: one is the need to know in advance the network connectivity, the other is to be higher in the process of positioning the node density[6].

APIT (Approximate Point-in-Triangulation Test). APIT algorithm with higher positioning accuracy is adapted to the complex geographical environment. APIT localization algorithm of unknown nodes in the process of implementation, first of all, by collecting the neighbor node identifier, the location information, the information such as the size of the transmitting power to determine whether the node is located in the different of beacon nodes within the triangle, and then statistics include the unknown node triangle area, and the intersection of the triangle area constitutes a polygon, the polygons, largely determine the unknown node area and narrow the scope of unknown node, finally calculated the center of mass of the polygon area, and the center of mass as the location of the unknown node, thus realize the unknown node location. APIT localization algorithm basic principle is shown in Fig. 2.

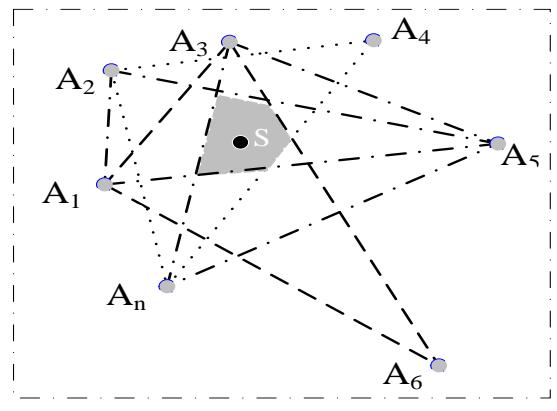

Figure 2. Diagram of APIT principle 
Convex Optimization Localization Algorithm. Convex Optimization is a kind of centralized positioning algorithm, the so-called centralized localization algorithm is relative to the case of a distributed localization algorithm, centralized positioning algorithm using concentrated thought, after collected all the sensor nodes effectively information unified positioning calculation, the extensibility of centralized localization algorithm is bad, signal transmission and processing more troublesome, will not be able to use flexible. Convex programming algorithm is shown in Fig. 3:

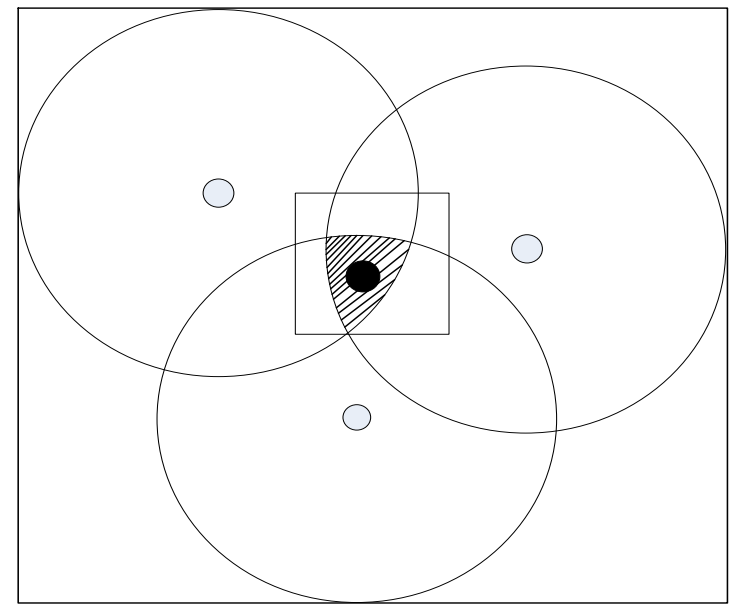

Figure 3. Diagram of convex optimization principle

The basic principle of unknown nodes according to the first communication radius and can communicate with the beacon node to establish its possible location area, when the exhaustion after these areas are filtered according to certain standards in these areas and position classification, finally determine a rectangle (as shown in Fig. 3 of the rectangular area of the shaded area), to calculate the mass center of the rectangle and place it as the coordinates of the unknown node, so as to realize the node localization.

MDS-MAP Localization Algorithm. MDS - MAP belongs to the centralized positioning algorithm, it is to use the node connectivity information between nodes generated by Dijkstra or Floyd algorithm distance matrix, then using Shortest Path Algorithm to get the location information between nodes. MDS to MAP the realization of the localization algorithm mainly divides into the following three steps:

(1) First of all, from the global perspective, according to the given node connectivity information between network topology connected graph is generated, and then through Shortest Path Algorithm to roughly estimate the distance between each pair of nodes, node distance matrix.

(2) The classic multidimensional scaling analysis technology was applied to the node distance matrix, calculated the entire network of $2 \mathrm{~d}$ or $3 \mathrm{~d}$ relative coordinate system, the coordinates of the relative coordinates.

(3) According to the position of the beacon node and beacon node of the node from the density of (2) the relative coordinate transformation, make it become the absolute coordinate system.

MDS - MAP algorithms assume that are connected between each pair of nodes, when the nodes density is small [7], not only the node positioning error is very big, the outside also can lead to many nodes can't locate. Only the network connectivity to a certain extent, all of the unknown node can realize positioning. Generally adopts the algorithm for node coordinates for the relative position coordinates, transformed into absolute coordinates to matrix operation, the relative coordinates of unknown nodes in the two-dimensional space into absolute coordinates generally requires at least three beacon node for auxiliary operation, in the three-dimensional space requires at least four beacon nodes, so the algorithm calculation and energy consumption are generally bigger.

All Range-Free Localization Algorithm Performance Comparison. Six range-free localization algorithms are compared in Table 1 
Table 1 Comparison of range-free algorithm

\begin{tabular}{|c|c|c|c|c|c|}
\hline Name & $\begin{array}{l}\text { Density of } \\
\text { Node }\end{array}$ & $\begin{array}{l}\text { Density of } \\
\text { Beacon Node }\end{array}$ & $\begin{array}{l}\text { Need for } \\
\text { additional } \\
\text { devices }\end{array}$ & $\begin{array}{l}\text { Beacon node } \\
\text { localization errors }\end{array}$ & $\begin{array}{l}\text { Positional } \\
\text { Accuracy }\end{array}$ \\
\hline $\begin{array}{l}\text { DV-HO } \\
n\end{array}$ & greater & greater impact & no & good & good \\
\hline Centroid & greater & greater impact & no & good & general \\
\hline Amorph & little impact & greater impact & yes & general & general \\
\hline APIT & greater & little impact & no & good & general \\
\hline Convex & little impact & little impact & no & good & better \\
\hline $\begin{array}{l}\text { MDS-M } \\
\text { AP }\end{array}$ & $\begin{array}{l}\text { greater } \\
\text { impact }\end{array}$ & greater impact & yes & general & good \\
\hline
\end{tabular}

\section{Summary}

Various localization algorithm has different application field, things are different for different localization algorithm to choose from, what kind of algorithm has no absolute advantage, in a certain occasion is applicable, but once application environment is changed, the performance of this algorithm is probably will change. In the specific application environment to consider the characteristics of the algorithm and the actual situation, to security and positioning of the various parameters. In addition, in different application should also consider the combined use of several kinds of algorithms, division for the same kind of environment, different areas for different localization algorithm, and then combine these algorithms, it is also one of the focuses of research in the future.

\section{References}

[1] ESTRIN D, GOVINDAN R, HEIDEMANN J. Next century challenges: scalable coordination in sensor networks [J]. Proceedings of the Fifth Annual International Conference on Mobile Computing and Networks (Mobi COM'99), Washington, USA, 1999: 263-270.

[2] AGRE J, CLARE L. An integrated architecture for cooperative sensing networks [J]. IEEE Computer Magazine, 2000, 33(5): 106-108.

[3] SAMI M L, JAMES M C. Time synchronization in wireless sensor networks: A survey[C]. IEEE Southeast Con 2010 (Southeast Con), 2010. 242-245.

[4] W.A Gardner, C. K Chen. Singal selective time difference of arrival estimation for Passive location. IEEE Transaction on Signal Processing, 2012, 2. 1168 1184.

[5] GÜVENC I, CHONG C C. A survey on TOA based wireless localization and NLOS mitigation techniques [J]. IEEE Communications Surveys \& Tutorials, 2009, 11(3): 107 123

[6] KANG HSEO G, LEE W J. Error compensation for css-based localization system[C]. Proceedings of the World Congress on Engineering and Computer Science, 2009.

[7] ANDREAS L, CHRISTIAN W. A comprehensive approach for optimizing To A-localization in harsh industrial environments[C]. Position Location and Navigation Symposium (PLANS), 2010 IEEE/ION, 2010. 516 525. 